\title{
Um Estudo sobre Características Extraídas de Imagens Infravermelhas para Detecção de Anormalidades de Mama
}

\author{
Lincoln F. Silva ${ }^{1}$, Roger Resmini ${ }^{1}$, Aristófanes C. Silva ${ }^{3}$, Anselmo C. Paiva ${ }^{4}$, \\ Renato S. Bravo ${ }^{2}$, Alair Augusto S. M. D. Santos ${ }^{2}$, \\ Débora C. Muchaluat-Saade ${ }^{1}$ e Aura Conci ${ }^{1}$ \\ ${ }^{1}$ Instituto de Computação - Universidade Federal Fluminense (UFF) \\ ${ }^{2}$ Centro de Ciências Médicas - Universidade Federal Fluminense (UFF) \\ ${ }^{3}$ Departamento de Informática - Universidade Federal do Maranhão (UFMA) \\ ${ }^{4}$ Departamento de Engenharia de Eletricidade - Universidade Federal do Maranhão (UFMA)

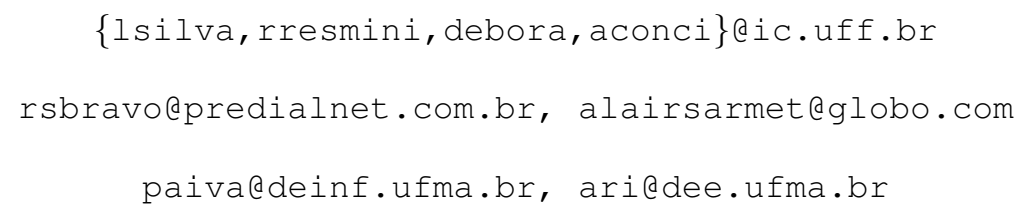

Abstract. Breast cancer is the second most common type of cancer worldwide. Diagnosis and treatment in early stages increase the chances of cure of the patient. The temperature of cancerous tissue is generally higher than that of healthy surrounding tissues, making thermography an option to be considered in screening strategies of this cancer type. This paper proposes a set of features extracted from images obtained by Dynamic Infrared Thermography in order to detect breast abnormalities, including cancer, using unsupervised machine learning techniques. Clustering validation Indexes are applied to evaluate formed groups of temperature time series for various $k$ value applied in the algorithm $k$-means, generating values treated as features. The analysis reveals that the Strehl index generates the most significant features.

Resumo. O câncer de mama é o segundo tipo de câncer mais comum no mundo. O diagnóstico e o tratamento em estágios iniciais aumentam as chances de cura da paciente. A temperatura de tecidos cancerosos é geralmente mais alta do que a de tecidos vizinhos saudáveis, tornando a termografia uma opção a ser considerada em estratégias de rastreamento deste tipo de câncer. Este artigo propõe um conjunto de características extraídas de imagens obtidas por Termografia Infravermelha Dinâmica com o objetivo de detectar anomalias da mama, entre elas o câncer, utilizando técnicas de aprendizagem de máquina não supervisionada. Índices de validação de agrupamento são aplicados para avaliar os grupos formados de séries temporais de temperatura para vários valor de $k$ aplicados no algoritmo $k$-means, gerando valores tratados como características. As análises revelam que o índice Streh gera as características mais significativas.

\section{Introdução}

O câncer de mama é o câncer mais comum entre as mulheres no mundo. Porém, quando identificado e tratado em estágios iniciais, esse tipo de câncer possui relativamente um 
bom prognóstico. Rastreamento é uma estratégia adotada por autoridades de saúde com o intuito de identificar mulheres que estão em estágios iniciais dessa doença de mama. Porém, todos os tipos de exames, incluindo a mamografia, considerada o padrão ouro na detecção de câncer, possuem suas limitações. A mamografia, por exemplo, possui alta taxa de classificação de falsos positivos, efetividade insuficiente em mamas densas e usa radiação ionizante para formar as imagens. Visto que a temperatura de tecidos cancerosos é geralmente maior do que a de tecidos saudáveis, a termografia tem sido considerada um método de rastreamento promissor para a detecção do câncer de mama, por gerar imagens que revelam a distribuição de calor sobre a superfície de ambas as mamas [Silva 2015].

A termografia é classificada basicamente em dois tipos: Termografia Infravermelha Estática (TIE) e Termografia Infravermelha Dinâmica (TID). As séries temporais usadas neste trabalho são obtidas a partir da TID, um método para monitorar a variação da temperatura da pele no tempo, após estresse térmico. O tipo de estresse térmico mais utilizado, em vários protocolos de execução da TID, é a aplicação de fluxo de ar direcionado às mamas utilizando um ventilador elétrico [Silva 2015]. O resfriamento das mamas, teoricamente, melhora o contraste térmico entre tecidos saudáveis e doentes na imagem, pois vasos sanguíneos gerados em função do tumor canceroso não possuem camada muscular e nem regulação neural como vasos embrionários [Amalu 2004]. Esses vasos são somente tubos endoteliais e portanto não contraem em resposta à estimulação simpática. Por essa razão, as regiões da mama com tumores cancerosos permanecem com temperatura quase inalterada, enquanto que a parte saudável da mama é resfriada [Amalu 2004]. A TID é mais rápida e mais robusta quando comparada à TIE, que requer condições rígidas de ambiente e tempo significativamente longo para aclimatação da paciente às condições da sala de exame. A TIE apenas registra a distribuição da temperatura na superfície da pele, não considerando sua variação no tempo. Por outro lado, a TID é menos dependente das condições e temperatura da sala de exame [Herman 2013]. Além disso, a Equação 1 [Gavriloaia et al. 2009] torna nítida a importância da variação da temperatura no tempo, onde $k$ é a condutividade térmica do tecido, $\rho c$ e $c_{b}$ são a densidade e o calor específico do sangue, $w_{b}$ é a taxa de perfusão do sangue $(\mathrm{ml} / \mathrm{s} / \mathrm{ml}), P$ é a taxa de geração de calor metabólico $\left(w / m^{3}\right), T_{b}$ é a temperatura do sangue arterial, e $T$ é a temperatura local do tecido. Observa-se nessa equação que existem termos de primeira derivada participal em relação ao tempo e de segunda derivada em relação a posição, mas não temperaturas constantes. Isso sugere a importância da variação da temperatura ao logo do tempo e, consequentemente, da análise dinâmica.

$$
\rho c \frac{\partial T}{\partial t}=\frac{1}{r^{2}} \frac{\partial}{\partial r}\left(r^{2} k \frac{\partial T}{\partial r}\right)+P+w_{b} c_{b}\left(T_{b}-T\right)
$$

Em um trabalho anterior, aplicamos classificadores tendo como entrada características extraídas de séries temporais de temperatura para identificar pacientes com câncer de mama. Os detalhes da metodologia estão em [Silva et al. 2015a]. Resumidamente, as imagens obtidas por TID de uma determinada paciente são primeiramente registradas. Em seguida, a região das mamas é dividida em quadrados pequenos e a temperatura máxima de cada um desses quadrados é observada em todas as imagens da paciente gerando, assim, as séries temporais de temperatura. Após, o algoritmo k-means é aplicado sobre tais séries formando $k$ grupos $(2 \leq k \leq 10)$. Índices de avaliação de agrupamento (ou validade de grupo) são aplicados para avaliar o resultado do agru- 
pamento produzido pelo algoritmo k-mean [MacQueen 1967] para cada valor de $k$. Os valores obtidos são tratados como características e submetidos à fase de seleção de características. Por fim, técnicas de aprendizagem de máquina são aplicadas para gerar o modelo de classificação tendo como entrada as características selecionadas. A evolução dessa metodologia nos últimos anos pode ser acompanhada em outros trabalhos do grupo em [Galvão et al. 2013] [Silva et al. 2014a][Silva et al. 2015b][Silva et al. 2016].

Neste artigo discutimos etapas específicas da metodologia para investigar os fatores que determinam a permanência de apenas alguns índices de avaliação de agrupamento no conjunto de características selecionadas. Para isso, cada índice foi analisado separadamente com o auxílio de gráficos para verificar o quanto o mesmo é discriminativo em relação a pacientes com e sem anormalidades de mama. Na análise de cada índice, todos os valores de $k(2 \leq k \leq 10)$ foram considerados, um por vez.

O restante deste artigo está organizado como segue. Na segunda seção, os principais trabalhos relacionados da literatura são discutidos. O metodologia proposta para análise das características extraídas é detalhado na terceira seção. Conclusões e trabalhos futuros são apresentados na última seção.

\section{Trabalhos relacionados}

Como uma revisão atualizada em TID de mama é apresentada em [Borchartt et al. 2013], esta seção focará somente em trabalhos computacionais mais recentes utilizando essa modalidade de exame.

No trabalho de Gerasimova et al. [Gerasimova et al. 2013], uma análise multifractal das séries temporais, geradas a partir da TID, foi executada para verificar a diferença de comportamento entre tecido com tumor maligno e tecido saudável. O método de máximo módulo da transformada de wavelet foi aplicado para caracterizar as propriedades multifractais de séries temporais oriundas de mamas cancerosas e saudáveis. Os autores concluíram que as propriedades escalares multifractais complexas, observadas em séries temporais sobre regulação automática (mamas saudáveis) são drasticamente alteradas na existência de doenças (mamas cancerosas). Nesse estudo, as duas mamas de 9 mulheres foram examinadas, 6 com câncer e 3 saudáveis. A metodologia conseguiu distinguir região com tumor e região saudável. Para mamas saudáveis, foi encontrada uma dimensão multifractal como característica de uma contínua mudança na forma da função de densidade de probabilidade de variação de temperatura através do tempo. Entretanto, séries temporais de temperatura da mama com tumor maligno mostraram estatísticas de variação de temperatura monofractal homogênea como evidência da perda de complexidade. As análises foram realizadas visualmente por meio de gráficos e tabelas.

Recentemente, Gerasimova et al. publicou outro trabalho [Gerasimova et al. 2014] usando uma base de dados maior, 33 pacientes com câncer de mama histopatologicamente confirmado e 14 voluntárias saudáveis para controle. Os achados reafirmaram os resultados de [Gerasimova et al. 2013].

Saniei et al. [Saniei et al. 2015] desenvolveram uma metodologia para análise de termografias obtidas por TID onde apenas duas imagens são usadas. Características são extraídas dos padrões vasculares e técnicas semelhantes às usadas para reconhecimento de impressões digitais são aplicadas para comparar tais padrões vasculares, dos termogramas 
antes e após o estresse térmico. Para quantificar o grau de similaridade entre os padrões vasculares nas duas imagens, um valor de pontuação de relacionamento é gerado por meio de uma expressão matemática e esses padrões serão considerados correspondentes se o valor de pontuação estiver abaixo de um determinado valor de limiarização, que é definido empiricamente. Segundo os autores, a metodologia alcançou sensibilidade de $86 \%$ e especificidade de $61 \%$. Foram usadas imagens de 50 pacientes, 25 com e $25 \mathrm{sem}$ câncer de mama.

\section{Metodologia proposta}

O fluxograma da metodologia proposta está na Figura 1. Primeiramente as séries temporais de temperatura são construídas, em seguida as características são extraídas e por último o estudo dessas características é realizado.

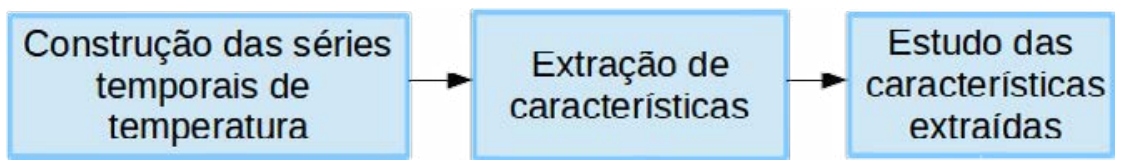

Figura 1. Fluxograma das etapas da metodologia proposta.

\subsection{Construção das séries temporais de temperatura}

Logo após a etapa de registro das imagens térmicas adquiridas por TID de uma paciente, detalhado em [Silva et al. 2015a], a construção das séries temporais de temperatura segue os seguintes passos:

1. a ROI (Region of Interest) no termograma, correspondendo aos pixels de cor branca na máscara (Figura 2 (a)), é divida em uma "malha" de quadrados $R_{k}$ de tamanho $11 \times 11$ pixels (Figura 2 (b)), com $k=1,2, \ldots, p$, onde $p$ é a quantidade de quadrados formados;

2. a temperatura mais alta de cada quadrado $R_{k}$ é observada em todos os vinte termogramas da paciente (Figura 2 (c)), produzindo a série temporal $S_{k}=$ $\left(t_{k, 1} ; t_{k, 2} ; \ldots ; t_{k, 20}\right)$, onde $t_{k, 1}$ é a temperatura mais alta da região quadrada $R_{k}$ no primeiro termograma da sequência, $t_{k, 2}$ é a temperatura mais alta da região quadrada $R_{k}$ na segunda imagem da sequência, e assim sucessivamente.
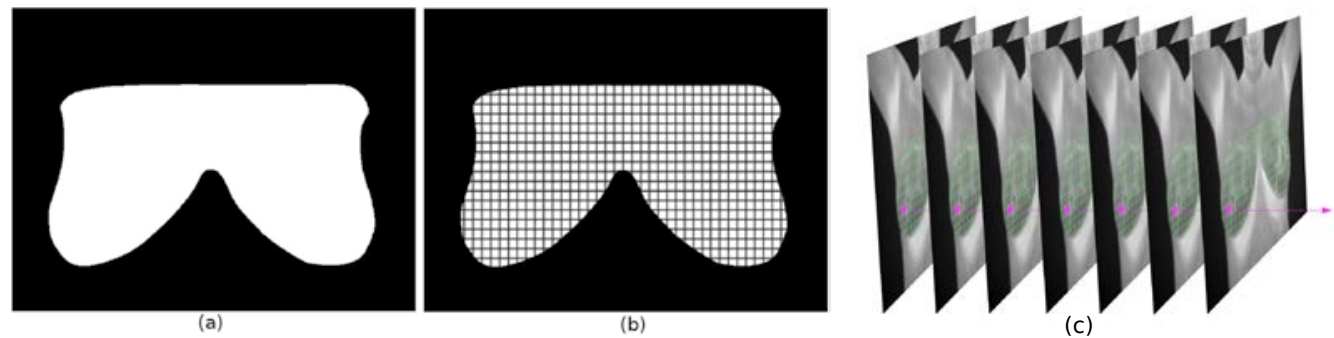

Figura 2. Máscara divida em uma "malha" de quadrados de tamanho 11x11 pixels e a contrução de uma série temporal de temperatura.

A Figura 3 mostra as séries temporais de temperatura de uma paciente saudável em (a) e as séries temporais do mesmo tipo de uma paciente com câncer de mama em 
(b). Em cada gráfico: o eixo $x$ representa o tempo (os 20 pontos de cada série); o eixo $y$ contém o índice de cada um das séries temporais de temperatura; e o eixo $z$ indica a temperatura em graus Celsius. É possível observar que existe um grupo de séries temporais com temperaturas maiores (cor vermelha) e com inclinação maior nos momentos iniciais da recuperação da temperatura, após o estresse térmico, para a paciente doente. $\mathrm{O}$ mesmo não é verdadeiro para as séries temporais da paciente saudável. As características extraídas buscam detectar as séries temporais de temperaturas que se destacam das demais para que seja possível indicar pacientes com anormalidade de mama.
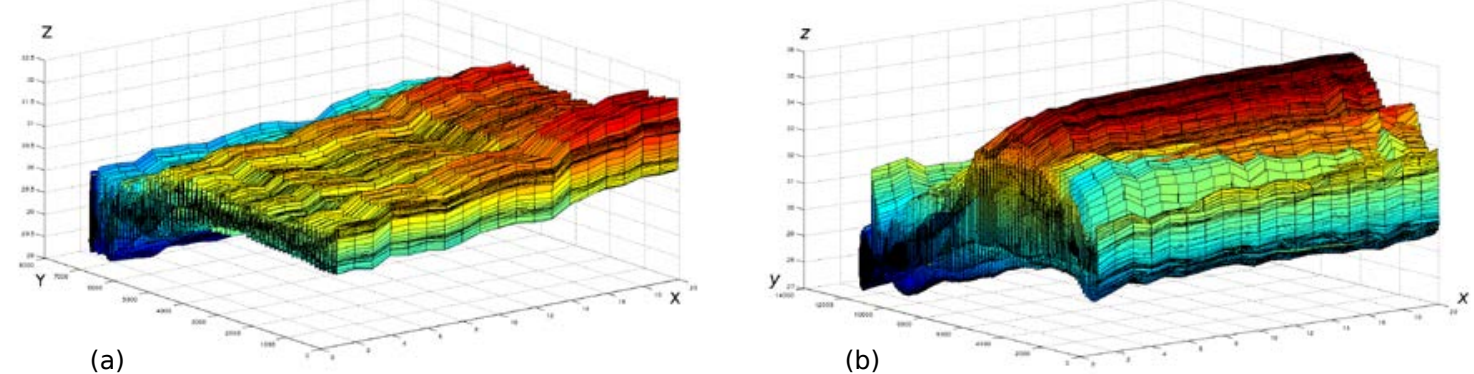

Figura 3. Em (a), sinais térmicos de uma paciente saudável, e em (b), sinais térmicos de uma paciente com câncer.

\subsection{Extração de características}

Dada uma paciente qualquer, as suposições são seguintes:

1. Se essa paciente for saudável, as séries temporais construídas a partir de cada ponto da mama são semelhantes (como na Figura 3 à esquerda), ou seja, possuem um grau alto de similaridade e então um agrupamento formado por tais séries temporais possui as seguintes características:

i) os grupos formados não são compactos;

ii) os grupos formados são próximos (são semelhantes) uns dos outros, em relação a uma medida de similaridade.

2. Por outro lado, se essa paciente for doente, para as séries temporais construídas a partir de cada ponto da mama, existe um grupo que se destaca dos demais por possuir séries temporais com comportamento diferente (como na Figura 3 à direita), e então um agrupamento formado por tais séries temporais possui as seguintes características:

i) os grupos formados são mais compactos, se comparados aos da suposição 1 ;

ii) os grupos formados são menos próximos (são menos semelhantes) uns dos outros, em relação a uma medida de similaridade, se comparados aos da suposição 1 .

Para medir a compacidade de cada grupo formado e a distância entre eles, dentro de um agrupamento formado pelo algoritmo $k$-means, medidas (índices) de validação de agrupamento foram aplicados na esperança de que essas medidas retornassem valores diferentes entre pacientes doentes e saudáveis. 
Na literatura existem vários índices de validação, porém neste trabalho foram aplicados apenas os direcionados a resultados de algoritmos de agrupamento rígido dos dados (cada elemento deve pertencer a um, e somente um, grupo). São eles: Silhouette [Rousseeuw 1987]; Davies-Bouldin [Davies and Bouldin 1979]; Calinski-Harabasz [Caliński and Harabasz 1974]; Dunn [Bolshakova and Azuaje 2003]; Krzanowski-Lai [Krzanowski and Lai 1988]; Hartigan [Hartigan 1985]; Homogeneity [Chen et al. 2002]; Separation [Chen et al. 2002]; Hubert-Levin (Cindex) [Bolshakova and Azuaje 2006]; e Strehl [Strehl and Ghosh 2000].

Para uma determinada paciente $i$, as séries temporais construídas na etapa anterior formam o conjunto, aqui denominado, $X_{i}$. Para separar tais grupos (de regiões saudáveis e de regiões com anomalias), uma técnica de aprendizagem de máquina não-supervisionada é aplicada por meio de um algoritmo de agrupamento. $\mathrm{O}$ algoritmo escolhido para essa tarefa é o k-means [Han and Kamber 2006]. O k-means é executado, tendo como entrada os elementos do conjunto $X_{i}$, nove vezes, ou seja, para cada valor de $k \in\{2,3, \ldots, 10\}$, gerando o conjunto de resultados de agrupamento $P_{i}=\left\{P_{i, 2}, P_{i, 3}, \ldots, P_{i, 10}\right\}$, onde $P_{i, 2}$ contém 2 grupos de séries temporais, $P_{i, 3}$ contém 3 grupos de séries temporais e assim sucessivamente até $P_{i, 10}$, que contém 10 grupos. A medida de proximidade utilizada no k-means é a distância Euclidiana.

O processamento ocorre na seguinte sequência:

1. o algoritmo $k$-means é executado para $k=2$ sobre o conjunto $X_{i}$, gerando o agrupamento $P_{i, 2}$;

2. os 10 índices de validação de agrupamento, citados anteriormente, são aplicados para medir a qualidade dos grupos formados no agrupamento $P_{i, 2}$, ou seja, para medir o quão compactos e bem separados os grupos são, e os resultados são armazenados no vetor $V_{i}$;

3. o valor de $k$ é atualizado para $k+1$ e os passos 1. e 2. são executados novamente. O processo descrito ocorre até o valor de $k$ igual a $10(k=10)$, e ao final o vetor $V_{i}$ contém 90 valores, ou seja, $V_{i}=\left(v_{i, 1}, v_{i, 2}, \ldots, v_{i, 90}\right)$. Todos os valores do vetor $V_{i}$ estão no intervalo $[0,1] \subset \mathbb{R}$, ou seja, $v_{i, j} \in[0,1] \subset \mathbb{R}, \forall j \in[2,10] \subset \mathbb{N}$.

\subsection{Estudo das características extraídas}

Em relação aos índices de validação de agrupamento, o mais discriminativo, ou seja, o que mais esteve presente entre as características selecionadas, na fase de seleção de características, foi o Strehl. Isso aconteceu para $k \in\{3,4,6,7,8,9,10\}$, onde $k$ é o número de grupos formados pelo $k$-means. Além dele, o índice Hubert-Levin (para $k \in\{3,8,10\}$ ), o índice Homogeneidade (para $k \in\{2,7\}$ ) e o índice Silhueta (para $k=3$ ) também estiveram presentes entre as características selecionadas. A seleção de características foi auxiliada pelo Auto-Weka [Thornton et al. 2013], que é uma ferramenta de mineração de dados usada para resolver o problema de seleção de algoritmos e otimização de parâmetros em tarefas de classificação. O método de seleção de características e seus parâmetros, recomendados pelo Auto-Weka, foram usados no WEKA para a seleção das características.

Uma investigação foi realizada para averiguar o motivo da superioridade do índice Strehl sobre os outros índices na tarefa de separar as pacientes doentes das saudáveis, o motivo que fez com que ele aparecesse tantas vezes no vetor das características selecionadas. Cada índice, para cada valor de $k$, foi analisado através de gráficos de linha 

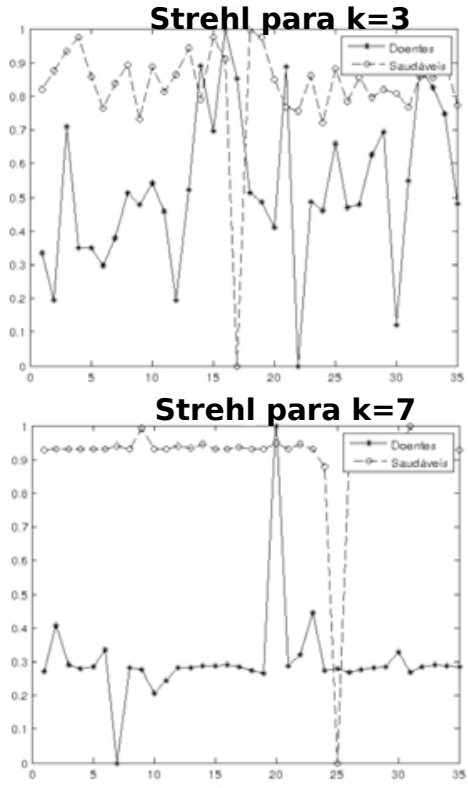
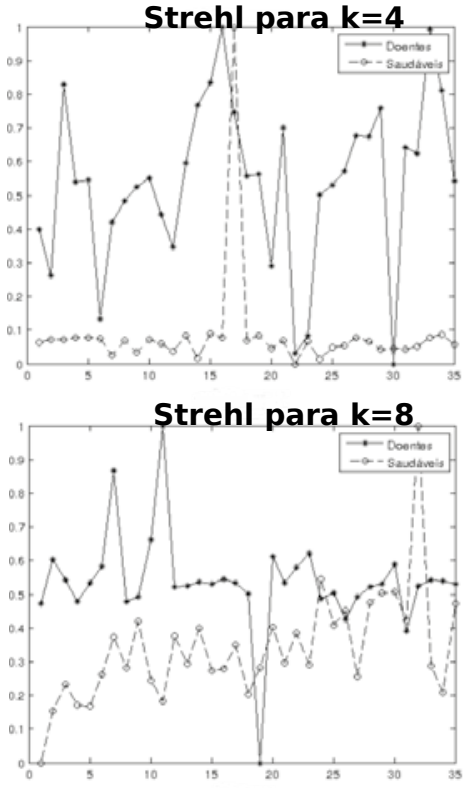

Strehl para $k=6$
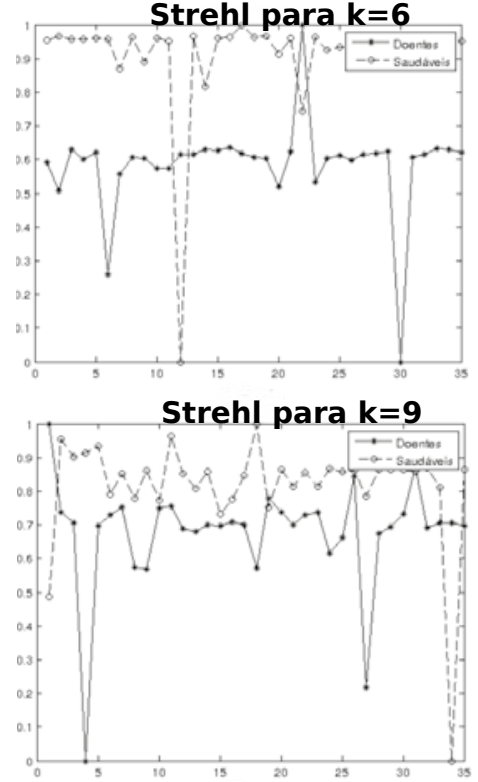

Figura 4. Cálculo do índice Strehl para $k=3,4,6,7,8,9$.

construídos a partir de pontos que representavam os valores do índice, calculado para cada paciente. Observando esses gráficos foi possível perceber que o índice Strehl de fato consegue praticamente separar os dois tipos de pacientes (doente e saudável), para quase todos os valores de $k$. Algo semelhante não aconteceu para os outros índices testados. Para ilustrar, a Figura 4 contêm os gráficos com o cálculo do índice Strehl para $k \in\{3,4,6,7,8,9\}$, ou seja, características que estiveram entre as selecionadas. Nos gráficos, o eixo horizontal representa o número (índice) das pacientes doentes e saudáveis, o eixo vertical é a escala de valores reais do índice Strehl, a linha contínua representa as pacientes doentes e a linha tracejada, as pacientes saudáveis. Assim, utilizando os valores desse índice quando ele é usado para avaliar os agrupamentos formados por 3, 4, 6, 7, 8 e 9 grupos de séries temporais é possível praticamente separar as pacientes doentes das saudáveis.

O índice Strehl utiliza as similaridades intragrupo e intergrupo para medir a qualidade dos $k$ grupos formados com os $n$ elementos de um conjunto de dados $X$. A similaridade intragrupo representa a similaridade média entre os elementos de um grupo $G_{i} \subset G$ ( $G$ é o resultado do agrupamento dos elementos de $X$ e $i \in 1,2, \ldots, k$ ) e é definida pela Equação 2:

$$
\operatorname{intra}\left(G_{i}\right)=\frac{1}{\left(g_{i}-1\right) g_{i}} \sum_{x, y \in G_{i}} d(x, y)
$$

onde $g_{i}$ é o número de elementos do grupo $G_{i}$ e $d(x, y)$ é a distância entre dois elementos de $G_{i}$. Essa equação não define a similaridade intragrupo para grupos vazios ou com um único elemento. Por definição, a similaridade intragrupo para grupo com um único elemento é igual a 1 (um).

A similaridade intergrupo entre grupos não vazios $G_{i}$ e $G_{j}$, com $i \neq j$ é definida 
pela Equação 3:

$$
\operatorname{inter}\left(G_{i}, G_{j}\right)=\frac{1}{g_{i} g_{j}} \sum_{x \in G_{i}, y \in G_{j}} d(x, y)
$$

onde $g_{i}$ e $g_{j}$ são o número de elementos do grupo $G_{i}$ e do grupo $G_{j}$, respectivamente.

O objetivo é maximizar a similaridade intragrupo e minimizar a similaridade intergrupo. Assim, a qualidade de agrupamento $G$ é definida como a razão entre o total das similaridades intragrupo e o total das similaridades intergrupo. O total das similaridades intragrupo é o somatório de cada uma das similaridades intragrupo ponderada por $g_{i}-1$, pois a auto-similaridade (o qual é sempre 1) não deve ser contabilizada. O total das similaridades intergrupo é obtido pelo somatório de cada similaridade $\operatorname{inter}\left(G_{i}, G_{j}\right)$ ponderada por $g_{i}$.

$$
\frac{\sum_{i=1}^{k} \frac{g_{i}-1}{n-k} \operatorname{intra}\left(G_{i}\right)}{\sum_{i=1}^{k} \sum_{j=i+1}^{k} \frac{g_{i}}{n} \operatorname{inter}\left(G_{i}, G_{j}\right)}
$$

O valor da razão 4 está dentro do intervalo $[0, \infty)$.

Sendo $G$ o resultado de um agrupamento dos elementos em $X$, a qualidade de $G$ é definida pela Equação 5:

$$
\Gamma(G)=1-\frac{(n-k) \sum_{i=1}^{k} \sum_{j=i+1}^{k} g_{i} \operatorname{inter}\left(G_{i}, G_{j}\right)}{n \sum_{i=1}^{k}\left(g_{i}-1\right) \operatorname{intra}\left(G_{i}\right)}
$$

onde $\Gamma(G) \in[0,1]$

A eficiência do índice Strehl é consequência de sua formulação, que pondera as similaridades intragrupo e intergrupos pelo tamanho de cada grupo envolvido no cálculo de tais similaridades, visto que, dependendo do tamanho das mamas da paciente, para cada uma (paciente) teremos mais ou menos séries temporais construídas. Isso não acontece para os demais índices, ou seja, as similaridades intragrupo e intergrupo são calculadas sem levar em conta o tamanho de cada grupo.

As imagens infravermelhas usadas neste trabalho são da Database for Mastology Research with Infrared Image - DMR-IR (http://visual.ic.uff.br/dmi/). Essa base de dados e o protocolo de aquisição das imagens são descritos em trabalhos anteriores do grupo [Silva et al. 2014b][Silva et al. 2013]. A aquição das imagens e o uso delas em pesquisas está registrado no Ministério da Saúde sob o número CAAE: 01042812.0.0000.5243.

\section{Conclusões}

Regiões da mama com câncer produzem séries temporais de temperatura com alteração de comportamento quando observados em função do tempo. Este trabalho realizou um estudo de características presentes na detecção dessas alterações usando aprendizagem de máquina não supervisionada. Os resultados mostraram que o índice Strehl, para vários valores de $k$ aplicado no algoritmo $k$-means, gera as características mais significativas na discriminação de mamas saudáveis e mamas com alguma anomalia. Como trabalho futuro, métodos de classificação serão testados apenas com as características geradas a partir do índice Strehl para indicar pacientes com alguma anormalidade de mama. Isso gerará uma diminuição de tempo e de processamento computacional, quando comparado ao nosso trabalho anterior, para realizar tal tarefa. 


\section{Referências}

Amalu, W. C. (2004). Nondestructive testing of the human breast: The validity of dynamic stress testing in medical infrared breast imaging. Engineering in Medicine and Biology Society, 1:1174-1177.

Bolshakova, N. and Azuaje, F. (2003). Cluster validation techniques for genome expression data. Signal Processing, 83(4):825-833.

Bolshakova, N. and Azuaje, F. (2006). Estimating the number of clusters in dna microarray data. Methods of Information in Medicine, 45(2):153-157.

Borchartt, T. B., Conci, A., Lima, R. C. F., Resmini, R., and Sanchez, A. (2013). Breast thermography from an image processing viewpoint: A survey. Signal Processing, 93:2785-2803.

Caliński, T. and Harabasz, J. (1974). A dendrite method for cluster analysis. Communications in Statistics, 3(1):1-27.

Chen, G., Jaradat, S. A., Banerjee, N., Tanaka, T. S., Ko, M. S. H., and Zhang, M. Q. (2002). Evaluation and comparison of clustering algorithms in anglying es cell gene expression data. Statistica Sinica, 12:241-262.

Davies, D. L. and Bouldin, D. W. (1979). A cluster separation measure. Pattern Analysis and Machine Intelligence, IEEE Transactions on, PAMI-1(2):224-227.

Galvão, S. S. L., Conci, A., Galvão, S. S. L., and Silva, L. F. (2013). Registro de imagens afim para o protocolo dinâmico de aquisição de imagens térmicas da mama. In $I V E n$ contro Nacional de Engenharia Biomecânica: ENEBI 2013, pages 158-159, Vitória, ES, Brazil.

Gavriloaia, G. V., Hurduc, A., Ghimigean, A.-M., and Fumarel, R. (2009). Spatialtemperature high resolution map for early cancer diagnosis. Proc. SPIE, 7171:71710W-71710W-8.

Gerasimova, E., Audit, B., Roux, S., Khalil, A., Gileva, O., Argoul, F., Naimark, O., and Arneodo, A. (2014). Wavelet-based multifractal analysis of dynamic infrared thermograms to assist in early breast cancer diagnosis. Frontiers in Physiology, 5(176).

Gerasimova, E., Audit, B., Roux, S. G., Khalil, A., Argoul, F., Naimark1, O., and Arneodo, A. (2013). Multifractal analysis of dynamic infrared imaging of breast cancer. EPL (Europhysics Letters), 104(6):68001-p1-68001-p6.

Han, J. and Kamber, M. (2006). Data Mining: Concepts and Techniques. Morgan Kaufmann Publishers, Burlington, MA 01803, USA, 2rd ed edition.

Hartigan, J. (1985). Statistical theory in clustering. Journal of Classification, 2(1):63-76.

Herman, C. (2013). The role of dynamic infrared imaging in melanoma diagnosis. Expert Review of Dermatology, 8(2):177-184.

Krzanowski, W. J. and Lai, Y. T. (1988). A criterion for determining the number of groups in a data set using sum-of-squares clustering. Biometrics, 44(1):23-34.

MacQueen, J. (1967). Some methods for classification and analysis of multivariate observations. In Proceedings of the Fifth Berkeley Symposium on Mathematical Statistics 
and Probability, Volume 1: Statistics, pages 281-297, Berkeley, Calif. University of California Press.

Rousseeuw, P. J. (1987). Silhouettes: A graphical aid to the interpretation and validation of cluster analysis. Journal of Computational and Applied Mathematics, 20(0):53-65.

Saniei, E., Setayeshi, S., Akbari, M. E., and Navid, M. (2015). A vascular network matching in dynamic thermography for breast cancer detection. Quantitative InfraRed Thermography Journal, 12(1):24-36.

Silva, L. F. (2015). Uma Análise Híbrida para Detecção de Anomalias da Mama usando Séries Temporais de Temperatura. PhD thesis, Universidade Federal Fluminense, Instituto de Computação, Niterói, RJ, Brasil.

Silva, L. F., Marques, R. S., Carvalho, G. S., Santos, M. L. O., Fontes, C. A. P., Santos, A. A. S. M. D., and Conci, A. (2013). Protocolo de captura de imagens térmicas da mama para construção de um banco público de exames. In IV Encontro Nacional de Engenharia Biomecânica: ENEBI 2013, pages 104-105, Vitória, ES, Brazil.

Silva, L. F., Olivera, G. O. S., Borchartt, T. B., Resmini, R., Santos, A. A. S. M. D., Fontes, C. A. P., Muchaluat-Saade, D. C., and Conci, A. (2015a). Uma análise hibrida para identificação de cancer de mama usando sinais térmicos. WIM-XV Workshop de Informática Médica-Anais CSBC.

Silva, L. F., Olivera, G. O. S., Galvão, S. S. L., Silva, J. B., Santos, A. A. S. M. D., Muchaluat-Saade, D. C., and Conci, A. (2014a). Análise de séries temporais de sinais térmicos da mama para detecção de anomalias. WIM-XIV Workshop de Informática Médica-Anais CSBC, pages 1818-1827.

Silva, L. F., Saade, D. C. M., Sequeiros, G. O., Silva, A. C., Paiva, A. C., Bravo, R. S., and Conci, A. (2014b). A new database for breast research with infrared image. Journal of Medical Imaging and Health Informatics, 4(1):92-100.

Silva, L. F., Santos, A. A. S., Bravo, R. S., Silva, A. C., Muchaluat-Saade, D. C., and Conci, A. (2016). Hybrid analysis for indicating patients with breast cancer using temperature time series. Computer Methods and Programs in Biomedicine, 130:142153.

Silva, L. F., Sequeiros, G., Santos, M. L., Fontes, C., Muchaluat-Saade, D. C., and Conci, A. (2015b). Thermal signal analysis for breast cancer risk verification. MEDINFO 2015: eHealth-enabled Health, Ebook Series: Studies in Health Technology and Informatics, 216:746-750.

Strehl, A. and Ghosh, J. (2000). Value-based customer grouping from large retail datasets. Proc. SPIE Conf. Data Mining Knowl. Discov., pages 33-42.

Thornton, C., Hutter, F., Hoos, H. H., and Leyton-Brown, K. (2013). Auto-WEKA: Combined selection and hyperparameter optimization of classification algorithms. Proceedings of the 19th ACM SIGKDD international conference on Knowledge discovery and data mining, pages 847-855. 